\title{
SURFACE OPTIMIZATION OF A SOCKET FOR A TRANSFEMORAL AMPUTEE THAT REDUCES THE STRESSES VARYING THE FRICTION COEFFICIENT
}

\author{
Vanessa Restrepo ${ }^{1}$, Junes Villarraga ${ }^{1}$, Jaime Velez ${ }^{1}$ \\ ${ }^{1}$ Department of Mechanical Engineering, University of Antioquia (vanerpo13@gmail.com)
}

\begin{abstract}
The shear stresses and pressures in the socket-stump interface were calculated for four transfemoral amputees, using the finite element method. A dynamic explicit model was developed for the simulation of the fitting procedure of the socket and the applying of the loads corresponding to the support phase during the gait, and a constitutive multilayer hyperelastic model was employed for the soft tissues (skin, fat and muscle) because of their large strains and displacements, and lineal isotropic models for the bone and socket.

In order to determine the influence of the friction coefficient on the socket-skin interface, its value was varied from a minimum value of 0.5 to a maximum of 1.0. Then, the zones where the greatest pressures and stresses occurred were identified, where the use of a horizontal strip was proposed with a variation of the friction coefficient from 0.2 to 0.6 , in order to observe its effect on the obtained stresses. The strip has a width of $10 \mathrm{~cm}$ and is located approximately $2.0 \mathrm{~cm}$ below the ischial support of all the individuals. It was observed that the pressure does not change significantly when varying the friction coefficient. However, the average shear stress tends to rise when increasing the friction coefficient, and after applying the strip the average shear stress reduced up to $25 \%$, while the pressure variation was not significant. Finally, the experimental validation of the behavior of the friction coefficient between the polypropylene and the skin was performed with a sclerometer. This experiment considered factors such as the patient's sweat, the amount of hair and the surface texture of the polypropylene coating, and it proved that the surface texture of the coating must be accounted for when measuring the friction coefficient.
\end{abstract}

Keywords: Transfemoral amputees, socket-stump interface, finiteelementanalysis, frictioncoefficient, stress distribution.

\section{INTRODUCTION}

A lower member prosthesis is an artificial device used to replace completely, or a portion of the missing lower member. It has different components according to the level of amputation, including a foot, a socket, and a joint between both, and in the case of transfemoral amputees, a prosthetic knee. The socket is the element of the prosthesis that is in direct contact with the patient, which is why its design is fundamental for the patient's perception of comfort when wearing the prosthesis [1]. 
A prosthesis is usually a handmade piece, and the knowledge and experience of the prosthetist is fundamental in the fabrication process, since there is no step by step manual which standardizes this procedure, and it varies according to each specialist and patient. Because of this it becomes difficult to standardize the final product and it is difficult to generalize the troubles of some amputees to all the other cases, since most of the problems are due to the prosthesis's shape.

It is estimated that 200-300 out of 100,000 individuals require amputation. This number is calculated considering that approximately $10 \%$ of the population has some sort of disability, and that $5 \%$ to $10 \%$ of the handicapped individuals are amputees. $85 \%$ of all amputations are due to peripheral vascular disease with or without diabetes, $10-12 \%$ are due to traumatic origin, and $3-5 \%$ to other complications [2].

The experimental methods available to measure pressures and stresses in the socketstump interface only provide approximations of localized points due to the complexities associated to the measurements. The Finite Element Method serves as a complement to the experimental measurements, and the measurements serve to validate the method [3][4]. In order to recreate as accurately as possible the stress and pressure distributions in the socket-stump interface of an amputee each study has a different emphasis according to certain simplifications and suppositions.

The objective of this investigation is to give continuity to a series of studies that have originated from a global project between the Universidad Nacional de Colombia Medellin Campus, and the Universidad de Antioquia, dealing with the study of the perception of comfort of transfemoral amputees with their prosthesis [5], and the analysis of the parameters that vary the stress distribution, such as the length of the stump [6] and the search of engineering solutions that improve the quality of life of these individuals.

\section{DEVELOPMENT AND METHODOLOGY}

In this investigation four individuals with transfemoral amputation were studied, each with a different age, gender, and amputation length. The subjects' data is presented in Table 1.

Table 1 : Patient Information

Code of the Subject Height [m] Body Weight [Kg] Stump Length[m]

\begin{tabular}{cccc}
\hline Indv 1 & 1.53 & 53.2 & 0.24 \\
Indv 2 & 1.75 & 75 & 0.24 \\
Indv 3 & 1.65 & 88.7 & 0.30 \\
Indv4 & 1.63 & 63.5 & 0.29 \\
\hline
\end{tabular}

In order to produce the CAD models a 3D scanner was used for the socket and the stump, and a series of tomographies were used to produce the geometry of the bone. This information was then imported into the SolidWorks 2010 software to be digitized. 
The stump was divided into three constitutive layers Figure 1. These are the skin, the fat, and muscle, in order to simulate the conditions as accurate as possible, and to analyze the influence of each layer on the final results. The skin and fat have an approximate average thickness of $2 \mathrm{~mm}$ and $4 \mathrm{~mm}$ respectively, and the remaining portion of the stump is muscle [7].

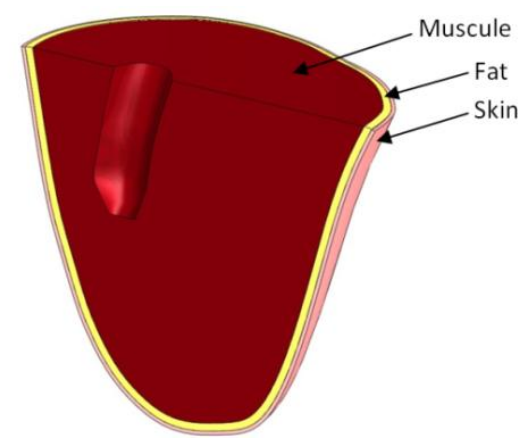

Figure 1 : Multilayer Stump

\subsection{FiniteElementModel}

The initial considerations taken into account when producing the model included the fitting of the socket by the amputee and its analysis under the most critical loads during the gait. In order to produce an accurate model of the fitting procedure, the model was divided into three analysis stages, which included the motion phase, the relaxation phase, and the load phase.

Motion: This stage accounted for the vertical motion of the socket during the fitting stage, from a configuration in which there is no contact with the stump to its final configuration in which it contains all of the soft tissue and bone.In this step, the amputees facilitate the fitting (sliding) of the socket using a thin sock or a liner, that reduces the friction coefficient, and therefore allows for a smoother fitting. Because this stage has specific characteristics that cause it to differ from the other two, it was considered as an independent stage. Also, the greatest relative movement of the socket with respect to the stump occurs during this stage.

During the motion step, the soft tissue must deform considerably in order to be contained by the socket which has a specific geometry. Figure 2 shows the fitting procedure of the socket.

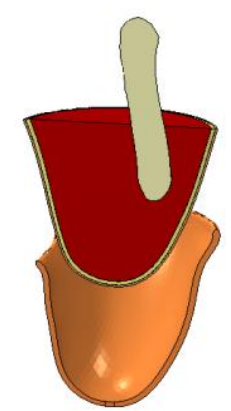

O Sec

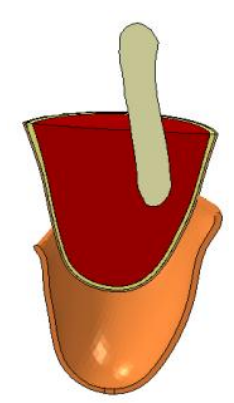

3 Sec

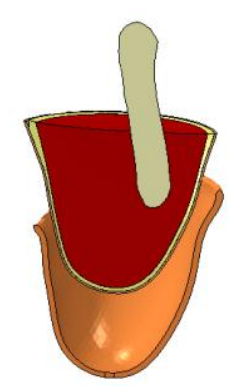

5Sec

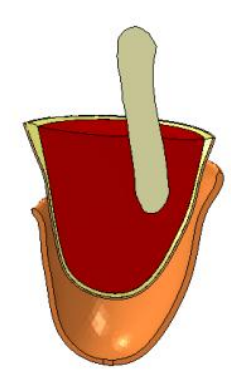

7 Sec

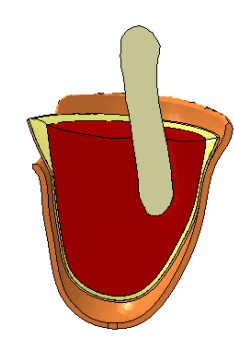

$10 \mathrm{Sec}$

Figure 2 : Fitting of the Socket 
Relaxation: The relaxation phase is considered as a transition phase between the motion phase (the socket's final configuration) and the exertion of the load. In this step, a three second interval was established to allow the soft tissue to adjust to the socket's geometry and to dissipate the changes occurring as waves or creases in the skin, product of the considerable deformations that occurred during the motion step.

Load: During this phase the most critical load of the gait phase is applied. This load is located on the rigid part of the socket, where it joins the rod of the thigh. It can reach magnitudes of up to $120 \%$ of the individual's body weight, and it is produced at the beginning of the initial support phase, and at the end of the initial and final support phases with a horizontal load representing $7 \%$ to $10 \%$ of the individual's body weight. This step is applied after the relaxation phase, and lasts for 0.1 seconds. In this phase, the relative motion between the socket and stump is not considered.

The main consideration accounted for when this investigation was performed was the fact that the soft tissue, such as the skin, fat and muscle were treated as hyperelastic, homogeneous and isotropic materials, with a density of $1000 \mathrm{Kg} / \mathrm{m}^{3}$ [8].Using the Mooney-Rivlin generalized deformation Equation 1, the constitutive parameters $C_{10}, C_{11}, D_{1}$ [7] were introduced into the ABAQUS V.6.9.2.software.

$$
w=c_{10}\left(I_{1}-3\right)+c_{11}\left(I_{1}-3\right)\left(I_{2}-3\right)-\frac{1}{D_{1}}(J-1)^{2}
$$

Where $\mathrm{I}_{1}, \mathrm{I}_{2}, \mathrm{~J}$ are invariable stresses of the main tensor and $\mathrm{c}_{10}, \mathrm{c}_{11}, \mathrm{D}_{1}$ are constants determined by experimentation.

The bone and the polypropylene socket were assumed as lineal, homogeneous and isotropic materials. The properties assigned to each of the materials are presented in Table 2 and Table 3.

Table 2 : Mechanical Properties of the Hyperelastic Materials (see [7])

\begin{tabular}{cccc}
\hline Soft Tissue & $\mathrm{C}_{10}(\mathrm{kPa})$ & $\mathrm{C}_{11}(\mathrm{kPa})$ & $\mathrm{D}_{1}\left(\mathrm{MPa}^{-1}\right)$ \\
\hline Skin & 9.400 & 82 & 0 \\
Fat & 0.143 & 0 & 70.20 \\
Muscle & 8.075 & 0 & 1.243 \\
\hline
\end{tabular}

Table 3 : Properties of the Elastic Materials

\begin{tabular}{cccccc}
\hline Element & Material & $\begin{array}{c}\text { Young's } \\
\text { Modulus }\end{array}$ & $\begin{array}{c}\text { Poisson's Coef- } \\
\text { ficient }\end{array}$ & Density & Reference \\
\hline Socket & Polypropylene & $1.5 \mathrm{GPa}$ & 0.3 & $800 \mathrm{Kg} / \mathrm{m}^{3}$ & {$[9]-[10]$} \\
Bone & Cortical Bone & $15 \mathrm{GPa}$ & 0.3 & $2000 \mathrm{Kg} / \mathrm{m}^{3}$ & {$[11]$} \\
\hline
\end{tabular}

Furthermore, tetrahedral four node C3D4 lineal elements and an automatic meshing algorithm were used, because the model's complexity does not allow for a structured mesh. In order to characterize the model as accurately as possible, the bone was fixed in the acetabulum, as shown in Figure 3, in order to restrict the movement and rotation in any direction. 
This fixture was added because in the initial support phase there is no relative movement of the acetabulum with respect to the hip, due to the effect of the muscles on the femur. This restriction allows the load transfer to the hip joint.

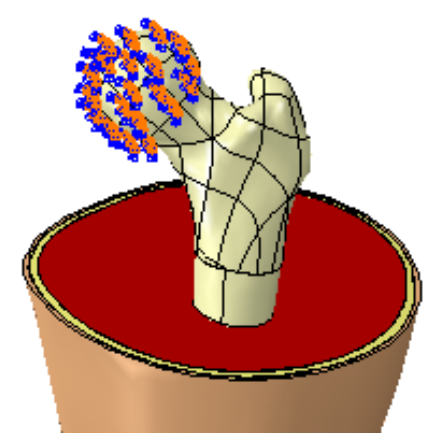

Figure 3 : Fixing of the Acetabulum

A Tie type restriction was applied to the skin-fat and fat-muscle interfaces, and to the muscle-bone interface, since this interface was assumed as adhered. The loads and torques that were applied in the linkage of the socket and the prosthesis [12] to perform the simulation were extracted from a previous study that extrapolated the static gait analysis of a human without disability to the gait of transfemoral amputee. The data is shown in Table 4.

Table 4 : Loads and Moments Applied to the Models

Code of the Subject Normal F [N] Shear F [N] Moment [N*m]

\begin{tabular}{llll}
\hline Indv1 & 567 & 207 & 100 \\
Indv2 & 807 & 295 & 172 \\
Indv3 & 961 & 352 & 167 \\
Indv4 & 682 & 249 & 119 \\
\hline
\end{tabular}

The load application point was established in the joint of the socket and the rod of the prosthesis that serves as a connection to the rest of the components. In this point the socket was considered as a rigid body, as shown in Figure 4.

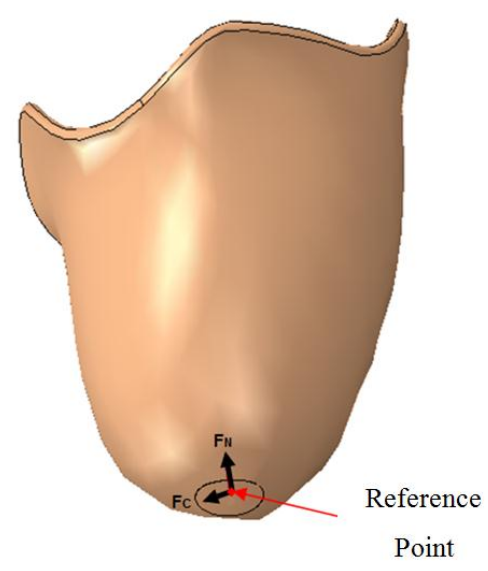

Figure 4 : Load Application in the Socket 


\subsubsection{Reference Models}

Different reference models were generated for each individual, varying the friction coefficient between the polypropylene and the skin from 0.5 to 1 . Upon completion of the simulations, a $10 \mathrm{~cm}$ wide horizontal high-stress strip was identified in all of the models, approximately $2 \mathrm{~cm}$ below the ischial support as shown in Figure 5.

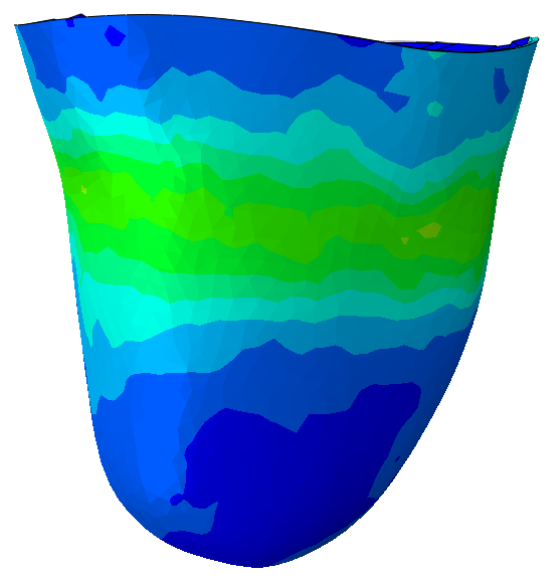

Figure 5 : Von Mises Stress Distribution

\subsubsection{Optimized Models}

After identifying the location of the strip a partition was made on the socket that allowed the modification of the friction coefficient in the area of interest (the strip), regardless of the global friction coefficient of the model.

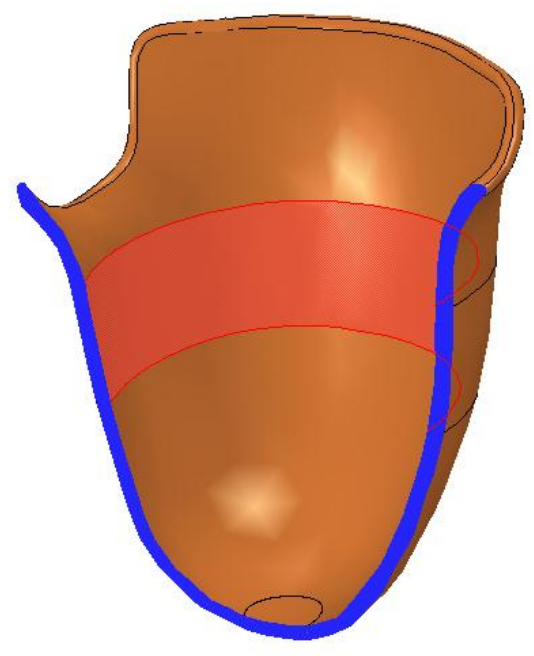

Figure 6 : Location of the Strip on the Socket 
Once the modification was performed to the socket (without altering the material), the global friction coefficient was set to 0.9 [7], and the friction coefficient of the red strip shown in Figure 6 was varied from 0.2 to 0.6 .

\subsection{Experimentation}

\subsubsection{Design of the Experiment}

To produce this work, a $2^{\mathrm{k}}$ factorial type experiment was performed, meaning that it consisted of 2 levels and " $\mathrm{k}$ " factors. The factors included hirsuteness, sweatiness, and texture, and the experiment was designed in the MINITAB 16 software. Table 5 shows the 16 configurations of the experiment and their measurement order in the $2^{3}$ factorial experiment with a replica ( -1 indicates a lack of the factor, and 1 indicates the presence of the factor).

Table 5 : Design of the Experiment

\begin{tabular}{cccc}
\hline Measurement & Hirsuteness & Sweatiness & Texture \\
\hline 1 & -1 & 1 & -1 \\
2 & -1 & -1 & -1 \\
3 & -1 & -1 & -1 \\
4 & 1 & -1 & -1 \\
5 & 1 & 1 & 1 \\
6 & 1 & 1 & -1 \\
7 & -1 & -1 & 1 \\
8 & 1 & 1 & 1 \\
9 & -1 & 1 & -1 \\
10 & 1 & -1 & 1 \\
11 & 1 & -1 & -1 \\
12 & -1 & 1 & 1 \\
13 & -1 & 1 & 1 \\
14 & 1 & -1 & 1 \\
15 & -1 & -1 & 1 \\
16 & 1 & 1 & -1 \\
\hline
\end{tabular}

\subsubsection{Considerations of the Factors}

Hirsuteness: In order to test the high hirsuteness levels the individual's right arm was left in original conditions, and to test the low levels the left arm was shaved.

Sweatiness: The high sweatiness levels were assessed with an artificial sweat solution prepared according to the NTC5221 standard, applying a thin layer (spray) between the skin and the indenter. In order to assess to low sweatiness levels the area was left clean and dry.

Texture: The coatings of the indenters were produced using a mold. For the high levels a $100 \%$ Nylon sock was employed, to simulate the fabrication process. This leaves a series of 
prints in the coating. The low texture levels were simulated leaving only the mold to prevent the markings from originating on the surface.

Using a surface roughness measuring device, as shown in Figure 7-a. (property of the Universidad Nacional de Colombia Sede Medellín Campus) it was possible to obtain the primary profiles of both coatings of the indenters that were used in the experiment, as shown in Figure 7-b. and Figure 7-c.

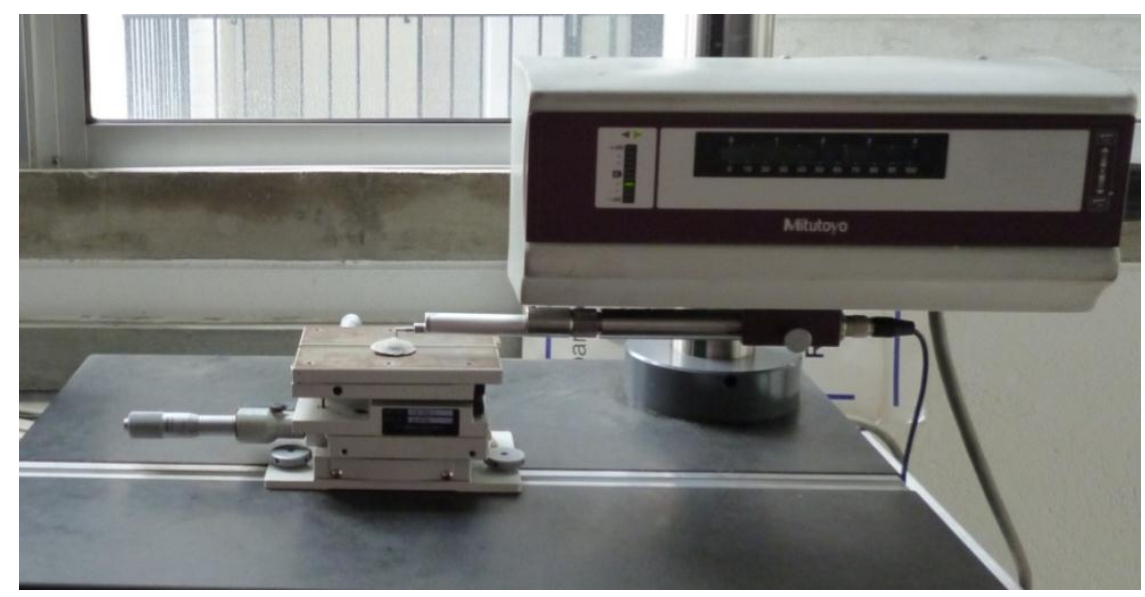

a.

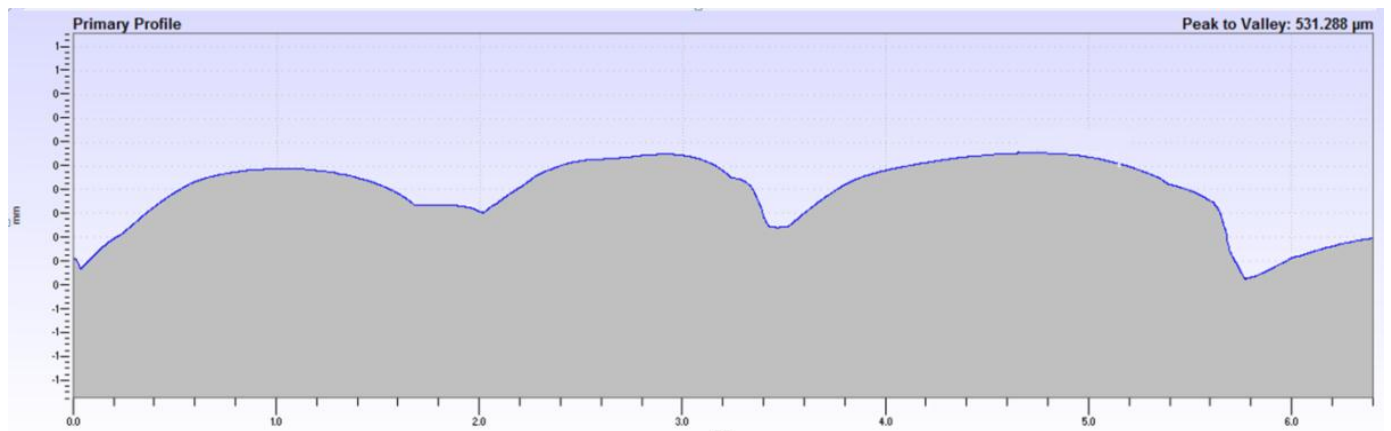

b.

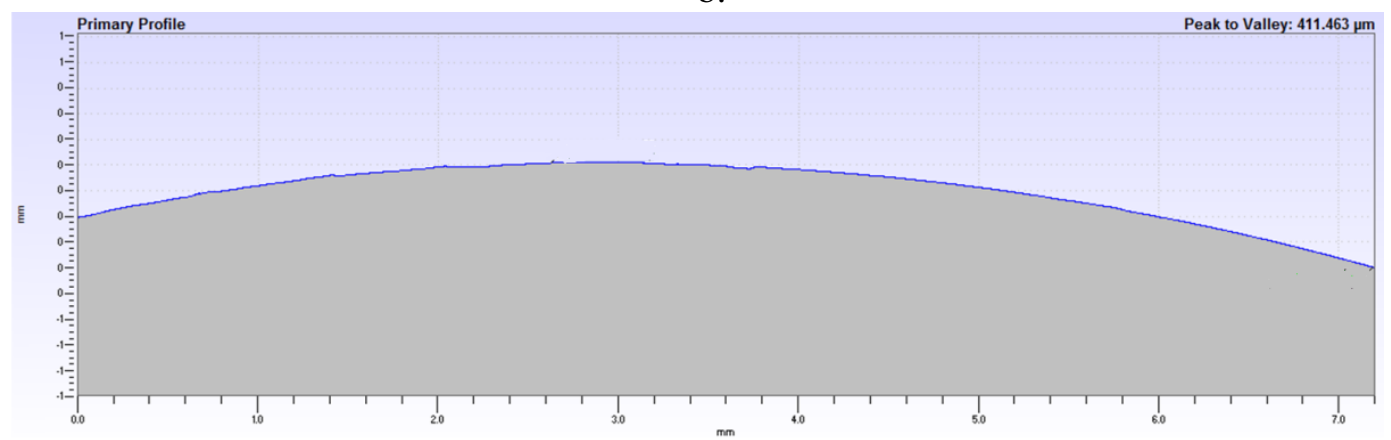

c.

Figure 7 : a. Surface Roughness Measuring Device, b. Primary Profile of the High Texture Coating, c. Low texture Coating

\subsubsection{Determining the Friction Coefficient of the Polypropylene-Skin Interface}

Once the experiment was organized, the most pertinent measurements were performed in a sclerometer (property of the Universidad Nacional Sede Medellín), as shown in Figure 8. 
The 16 measurements were performed on the same individual in order to block any factor that could affect the friction coefficient, such as the tone and moisture levels of the skin, the body mass index, the individual's physical activity, etc. All measurements were performed on the same day, to account for the factors that could not be controlled, including the relative humidity and temperature. Also, the equipment was calibrated to ensure that the data was as precise as possible.

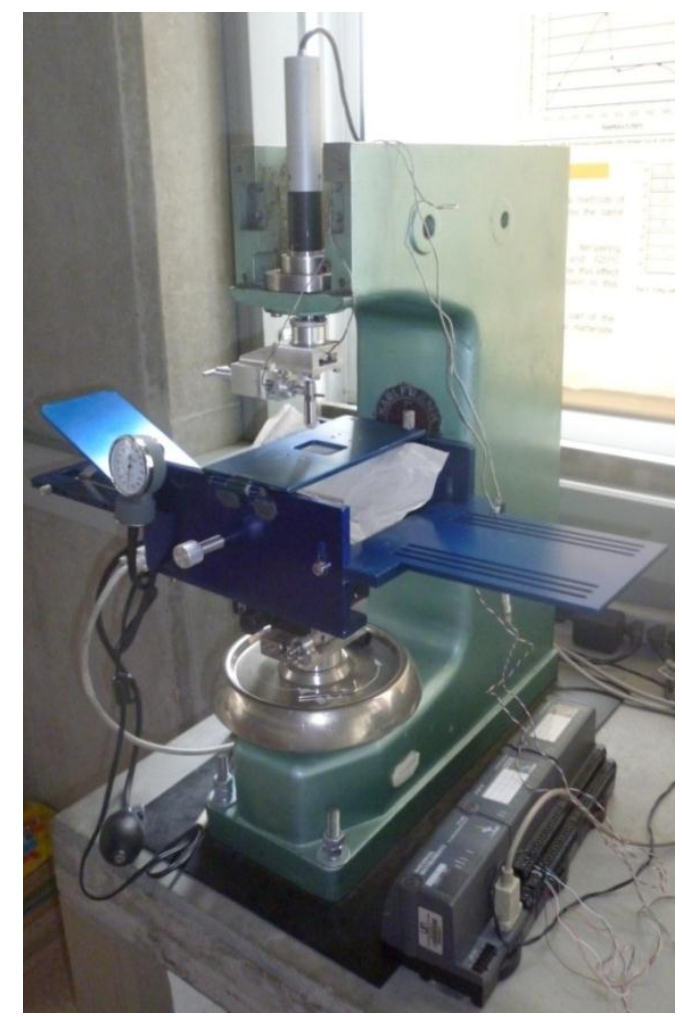

Figure 8 : Sclerometer

Figure 9 shows the typical behavior of the 16 measurements, where the friction coefficient increases proportionally to the displacement until it reaches the maximum value, and decreases slightly until it reaches a stable value. This means that while the friction coefficient increases lineally, there is no relative movement between the indenter and the skin. Once it reaches the maximum value, the indenter begins moving and the friction coefficient decreases because it surpasses the initial resistance of the skin, and when the indenter moves, the coefficient reaches a stable value, which is the objective of the measurement. 


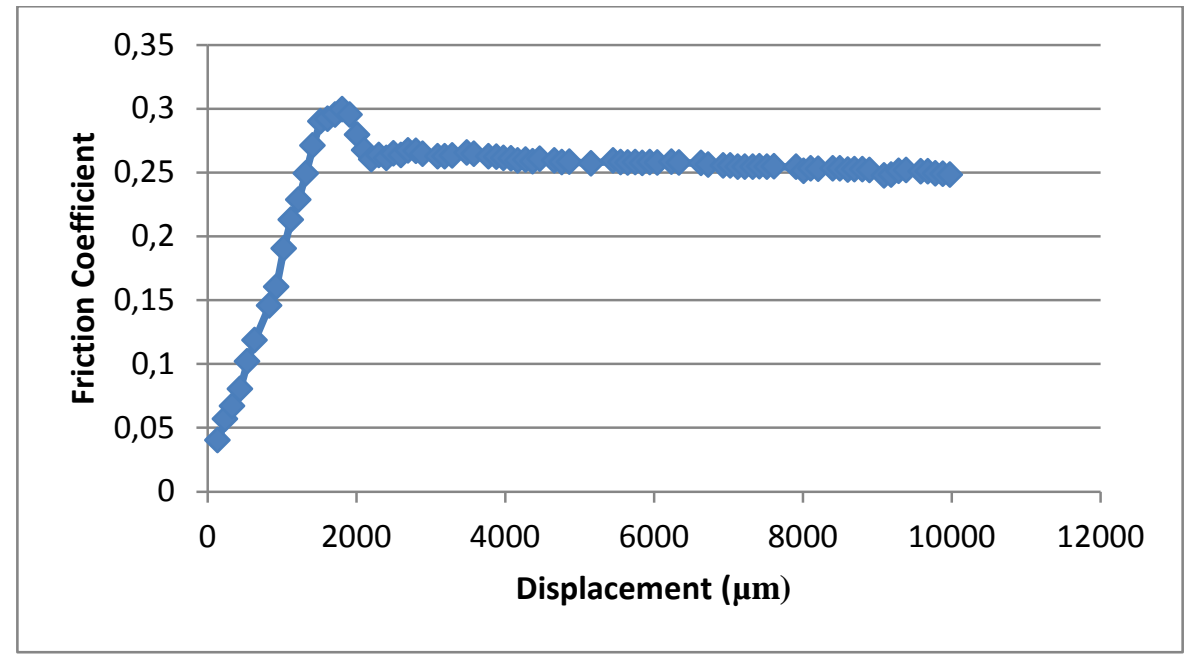

Figure 9: Typical Graph of the Friction Coefficient Vs Displacement

The friction coefficient was identified for all 16 measurements. These values are shown in Table 6.

Table 6 : Values of the Friction Coefficient

\begin{tabular}{ccccccc}
\hline Measurement & $\begin{array}{c}\text { Friction Coef- } \\
\text { ficient }\end{array}$ & Replica & $\begin{array}{c}\text { Friction Coef- } \\
\text { ficient }\end{array}$ & Hirsuteness & $\begin{array}{c}\text { Factors } \\
\text { Sweatiness }\end{array}$ & Texture \\
\hline 1 & 0.361 & 9 & 0.240 & -1 & 1 & -1 \\
2 & 0.522 & 10 & 0.545 & -1 & -1 & -1 \\
3 & 0.349 & 11 & 0.345 & 1 & -1 & -1 \\
4 & 0.229 & 12 & 0.232 & 1 & 1 & 1 \\
5 & 0.188 & 13 & 0.186 & 1 & 1 & -1 \\
6 & 0.266 & 14 & 0.396 & -1 & -1 & 1 \\
7 & 0.242 & 15 & 0.270 & 1 & -1 & 1 \\
8 & 0.261 & 16 & 0.250 & -1 & 1 & 1 \\
\hline
\end{tabular}

The analyses of the data obtained in MINITAB 16 are displayed in Table 7, and show the importance of the factors in the experiment. In order for a factor to be significant, the p-value (p) must be below 0.005 . Table 7 also accounts for $90.56 \%$ of the data $\left(\mathrm{R}^{2}\right)$.

Table 7 : P-values of the Factors

\begin{tabular}{cc}
\hline Factor & P-value $(\mathrm{p})$ \\
\hline Hirsuteness & 0.002 \\
Sweatiness & 0.001 \\
Texture & 0.012 \\
Hirsuteness*Sweatiness & 0.212 \\
Hirsuteness*Texture & 0.058 \\
Sweatiness*Texture & 0.012 \\
Hirsuteness*Sweatiness*Texture & 0.804 \\
\hline
\end{tabular}


The Hirsuteness, Sweatiness, Texture and the combination between the sweatiness and texture are significant and affect the behavior of the friction coefficient between the polypropylene and the skin.

\section{RESULTS AND ANALYSIS}

Once all the simulations for the reference models were completed and optimized with the respective variations of the friction coefficient between the polypropylene and the skin, the results were obtained for different variables. Considering the scope of this investigation, only the changes in the shear stresses and the pressures in the socket-stump interface were analyzed.

\subsection{Reference Model}

The objective of using this model is to locate an area where the highest stresses are concentrated, and to dwell on the incidence of the variation of the friction coefficient on these.

\subsubsection{Stress Distribution}

The most relevant stresses, considering the scope of this work are the pressures and the shear stresses 1 and 2. Once the simulations were completed their distributions were obtained by varying the friction coefficient. In the following figures the pressure and shear stress distributions 1 and 2 can be observed for the different subjects. These were obtained by varying the friction coefficient between the stump and the socket from 0.5 to 1.0 . In Figure 10 a $10 \mathrm{~cm}$ wide horizontal strip can be identified below the ischial support where the pressures and shear stresses are concentrated. The magnitudes shown in the figure are comparable to those reported in similar works [5]. 


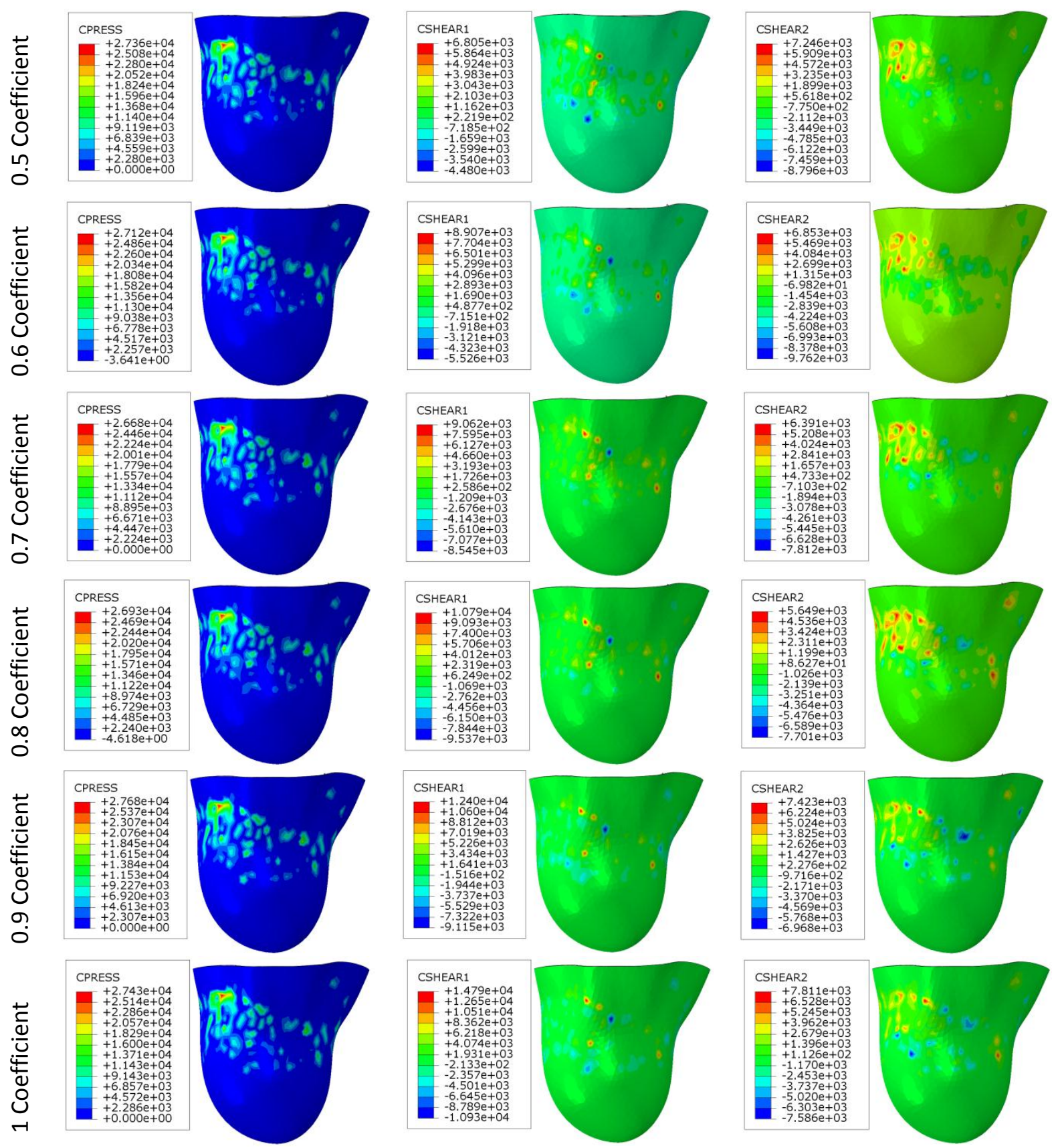

Figure 10 : Stress Distribution in the Reference Model for Individual 1

The shear stresses present a high stress concentration gradient which is not convenient because it increases the risk of generating injuries in the soft tissue. This stress concentration gradient may be attributed to the use of hyperelastic materials in the simulation, which better accommodate to the socket's geometry.

Figure 11 shows the behavior of the average shear stress after varying the friction coefficient, which tends to be lineal, and proportional to the friction coefficient. This behavior is similar to the one reported for a transtibial amputee [13][14]. 


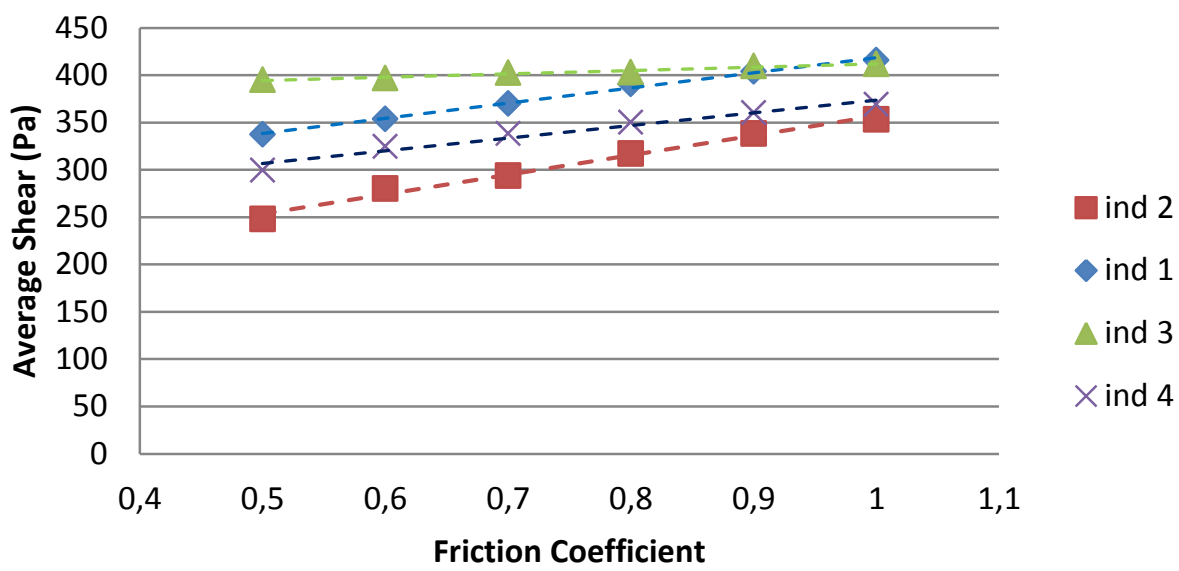

Figure 11 : Average Shear Stress Vs Friction Coefficient (Reference Model)

The average pressures did not present a behavior similar to that of the shear stresses. Figure 12 shows a more stable tendency, and does not present a significant change when varying the friction coefficient. A behavior different to the one presented in this work is reported; however the model used was much simpler [13], from a transtibial amputee, and the soft tissues were treated as elastic.

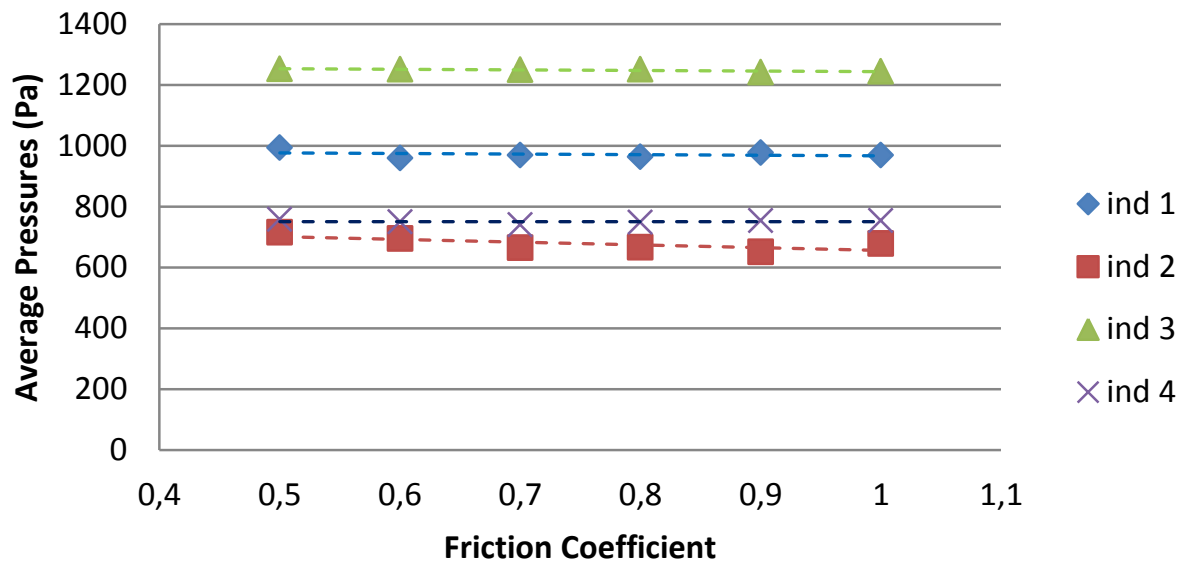

Figure 12: Average Pressures Vs Friction Coefficient (Reference Model)

\subsection{Optimized Model}

The produced socket was modified by altering the friction coefficient within the previously mentioned strip from 0.2 to 0.6 , while leaving the global friction coefficient in a set value $(0.9)$ 


\subsubsection{Stress Distribution}

Figure 13 shows the stress distribution on the skin, when varying the friction coefficient on the strip. The stress distribution was analyzed for the pressure, the shear stress 1 , and the shear stress 2 .
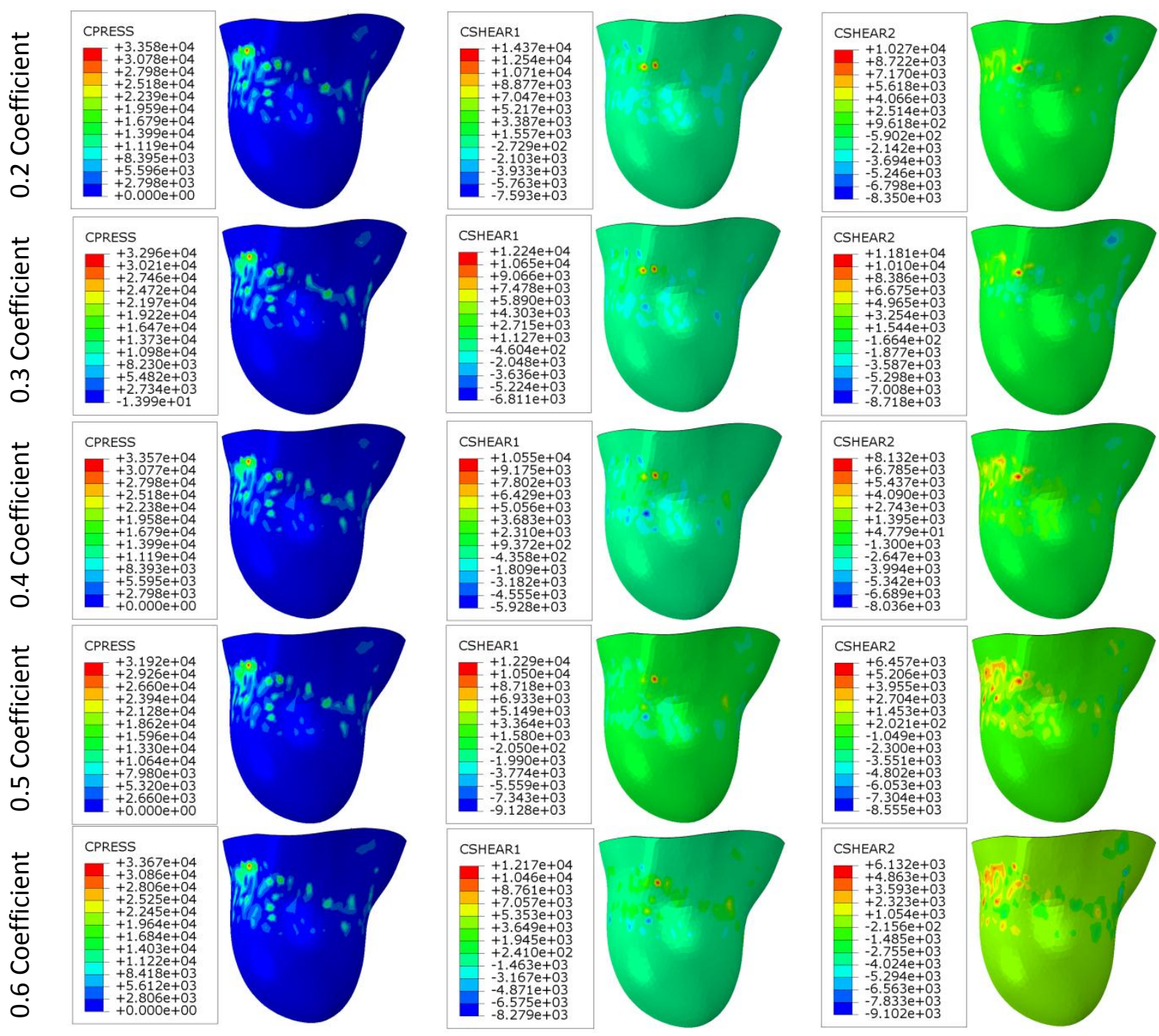

Figure 13 : Stress Distribution in the Optimized Model for Individual 1

The results obtained for the optimized model show similar tendencies to those obtained in the reference model, since shear stresses and pressure concentrations are present in the same place, generating a horizontal strip below the ischial support.

Figure 14 shows the same behavior identified in Figure 11 which corroborates the concept that the friction coefficient directly affects the values of the resulting shear stresses. 


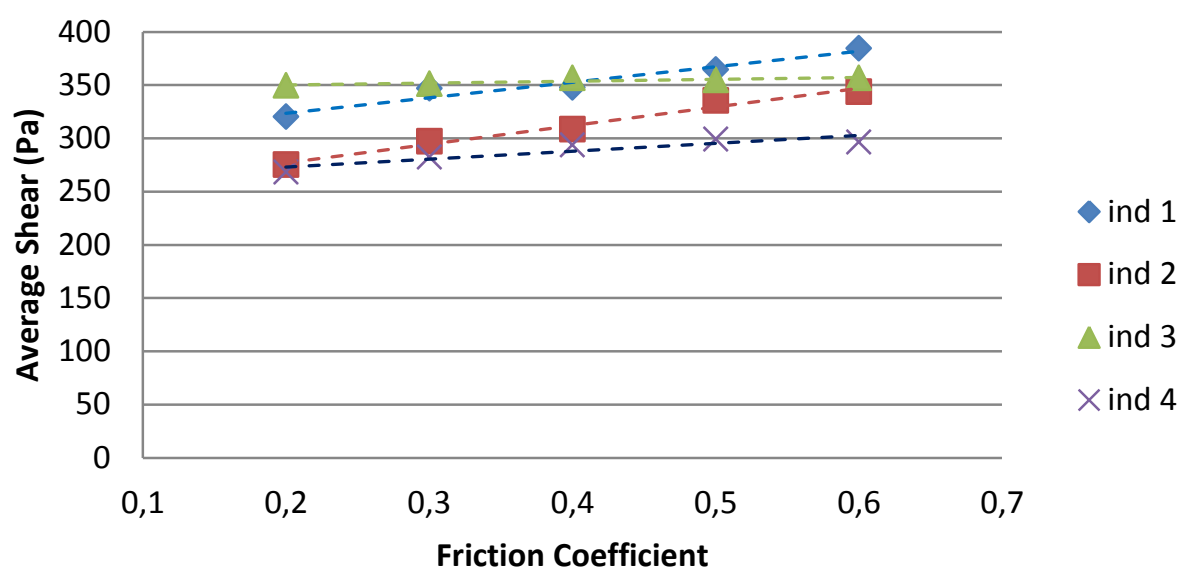

Figure 14 : Average Shear Stress Vs Friction Coefficient (Optimized Model)

Figure 15 shows the same behavior for the average pressures when modifying the friction coefficient. As in the reference model, a negligible variation occurred.

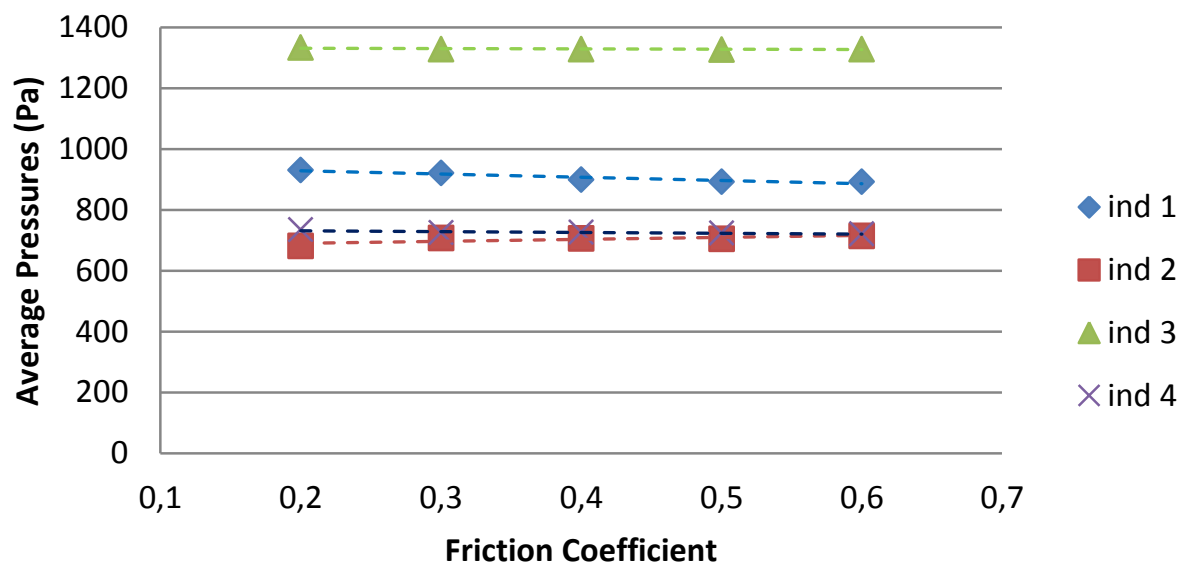

Figure 15 : Average Pressures Vs Friction Coefficient (Optimized Model)

Upon analyzing the results, the optimized model with a friction coefficient in the strip of 0.2 , and a global friction coefficient of 0.9 was selected as the most adequate, since this one produced the lowest values for the resulting shear stress. This model was compared to the model without the strip with an assigned friction coefficient of 0.9 to the entire socket-stump interface. The results (pressures and shear stresses) are presented in Table 8.

Table 8 : Stress Reduction Percentage

\begin{tabular}{ccc}
\hline Code of the Subject & Average Pressure $(\%)$ & Average Shear $(\%)$ \\
\hline Indv 1 & 4,75 & 20,69 \\
Indv2 & 4,58 & 18,67 \\
Indv 3 & 7,39 & 14,65 \\
Indv 4 & 2,68 & 25,61 \\
\hline
\end{tabular}




\section{CONCLUSIONS}

The selection of the soft tissues (skin, fat and muscle) as hyperelastic materials proved to be an asserted procedure, as these behaved like the real materials. The results are strictly comparable to those found in similar works; also the deformation process of the real tissue is physically compatible to the one observed in the simulation.

A horizontal strip was identified below the ischial support, where the pressures and shear stresses concentrate. These large stress concentrations occurred because the soft tissues were treated as hyperelastic materials, since these duplicate more effectively the geometry of the socket when they deform.

It was demonstrated that the friction coefficient between the polypropylene and the skin directly affects the value of the shear stresses, upon which the socket was modified by reducing the friction coefficient in the proposed horizontal strip. When the shear stresses are decreased, the risk of soft tissue damage is reduced.

The multilayer model is the most adequate to mimic the stress distribution in the stump with respect to the entire model, since the physical properties of the soft tissues are differentiated. This allows the simulation to deform in a way similar to the real phenomenon.

The friction coefficient between the polypropylene and the skin was modified by varying the surface texture of the coatings. From the results of the analysis it can be concluded that the texture indeed affects the average value of the friction coefficient. Therefore, a detailed study is proposed which deals with the influence of the tribological parameters, such as $\mathrm{Ra}$ and $\mathrm{Rq}$ measured on the internal surface of the socket on the friction coefficient.

The average shear stresses were reduced up to $25 \%$ after varying the friction coefficient on the strip. This proves that when changing the surface texture on the proposed strip a considerable improvement of the stress distribution can be obtained.

The models developed serve as a basis for future studies with more complex models that account for the interaction between the muscle and the bone, and simulate the stress distribution in the stump when negative pressure is applied on the sockets with a suction valve.

\section{Acknowledgements}

The authors would like to thank the Universidad de Antioquia for the technical support provided and the use of equipment, licenses and infrastructure, and the Universidad Nacional de Colombia Medellin Campus for the access to their measuring equipment andlicenses.

\section{REFERENCES}

[1] Meulenbelt H. E.,Geertzen J. H., Jonkman M. F., y Dijkstra P.

U.,"Determinantsofskinproblemsofthestump in lower-limbamputees", ArchPhysMedRehabil, vol. 90, $\mathrm{n}^{\circ} .1,74-81,2009$.

[2] «WHO | Disabilityandrehabilitation: WHO actionplan 2006-2011», WHO. [Online]. Available: http://www.who.int/disabilities/publications/action_plan/en/index.html. [Accessed: 16-abr-2012]. 
[3] Zhang M. y Roberts C., "Comparisonofcomputationalanalysiswithclinicalmeasurementofstressesonbelow-knee residual limb in a prosthetic socket", MedEngPhys, vol. 22, n . 9, 607-612, 2000.

[4] Manschot J. F. y Brakkee A. J.,"The measurementandmodellingofthemechanicalpropertiesofhumanskin in vivo--II. The model", J Biomech, vol. 19, $n^{\circ} .7,517-521,1986$.

[5] Ramírez J. F., "Nivel de Confort y Distribución de Esfuerzosenlalnterfaz Socket - Muñónen Amputados Transfemorales", TesisDoctoral, Universidad Nacional de Colombia, Medellín, Colombia, 2011.

[6] Restrepo V., "Optimización Superficial de un Socket para Amputado Transfemoral que DisminuyalosEsfuerzos Variando el Coeficiente de Fricción", Tesis de Pregrado, Universidad de Antioquia, Mdellín, 2012.

[7] Portnoy S.,Siev-Ner I.,Yizhar Z.,,Kristal A.,Shabshin N., y GefenA., "Surgicalandmorphologicalfactorsthataffectinternalmechanicalloads in soft tissuesofthetranstibialresiduum", Ann BiomedEng, vol. 37, $\mathrm{n}^{\circ} .12,2583-2605,2009$.

[8] ZhengY. P., MakA. F., yLeung A. K., "State-of-the-artmethods for geometricandbiomechanicalassessmentsof residual limbs: a review", J Rehabil Res Dev, vol. 38, nº. 5,487-504, 2001.

[9] Zhang M.yMakA. F., "A finiteelementanalysisoftheloadtransferbetweenanabove-knee residual limband its prosthetic socket-roles of interface frictionand distal-endboundaryconditions", IEEE TransactionsonRehabilitationEngineering, vol. 4, $\mathrm{n}^{\circ}$. 4, 337-346, 1996.

[10] Lee W. C. C.,Zhang M.,Jia X., y CheungJ. T. M., "Finiteelementmodelingofthecontact interface betweentrans-tibial residual limbandprosthetic socket", MedEngPhys, vol. 26, n. 8, 655-662, 2004.

[11] Duchemin L.,Bousson V.,Raossanaly C.,Bergot C.,Laredo J. D., SkalliW., y MittonD., "Predictionofmechanicalpropertiesof cortical bonebyquantitativecomputedtomography", MedEngPhys, vol. 30, $n^{\circ} .3,321-328,2008$.

[12] Vélez J. A. y Piedrahita M., "Análisis de la influencia delporcentajedelmiembro residual enladistribución de esfuerzos y presionesenlainterfaz socket-muñón", Tesis de pregrado, Universidad de Antioquia, Medellín, Colombia, 2011.

[13] Zhang M., LordM.,Turner-Smith A. R., yRoberts V. C., "Developmentof a non-linear finiteelementmodellingofthebelow-kneeprosthetic socket interface", MedEngPhys, vol. 17, $n^{\circ}$. 8, 559-566, 1995.

[14] Zhang M.,MakA. F., y Roberts V. C., "Finiteelementmodellingof a residual lower-limb in a prosthetic socket: a surveyofthedevelopment in thefirstdecade", MedEngPhys, vol. 20, $n^{\circ} .5,360-373$, 1998.

[15] Perry J., k S. T., y Davids J. R., "GaitAnalysis: Normal andPathologicalFunction", JournalofPediatricOrthopaedics, vol. 12, no. 6, 1992.

[16] Zhang M., Turner-Smith A. R., Roberts V. C., yTanner A., "Frictionalactionatlowerlimb/prosthetic socket interface", MedEngPhys, vol. 18, no. 3, 207-214, 1996.

[17] Appoldt F. A., Bennet L., y Contin R., "TangentialPressureMeasurements in Above-KneeSuction Sockets", SchoolofEngineeringand Science New York University. 1970.

[18] Williams R. B.,Porter D., Roberts V. C., yRegan J. F., "Triaxial force transducer for investigatingstressesatthestump/socket interface", MedBiolEngComput, vol. 30, no. 1, 89-96, 1992.

[19] Sanders J. E.,Daly C. H., y BurgessE. M., "Interface shearstressesduringambulationwith a belowkneeprostheticlimb", J Rehabil Res Dev, vol. 29, no. 4, 1-8, 1992.

[20] Silver-Thorn M. B.,Steege J. W., y ChildressD. S., "A reviewofprosthetic interface stress investigations", J Rehabil Res Dev, vol. 33, no. 3, 253-266, 1996.

[21] Colombo G.,Filippi S., Rizzi C., y RotiniF., "A new design paradigm for the developmentofcustom-fit soft sockets for lowerlimbprostheses", Computers in Industry, vol. 61, no. 6, 513-523, 2010.

[22] Huiskes R. y ChaoE. Y.,"A surveyoffiniteelementanalysis in orthopedicbiomechanics: thefirstdecade", J Biomech, vol. 16, no. 6, 385-409, 1983.

[23] Zachariah S. G. y SandersJ. E., "Finiteelementestimatesof interface stress in thetranstibialprosthesisusing gap elements are differentfromthoseusingautomatedcontact", J Biomech, vol. 33, no. 7, 895-899, 2000.

[24] Lee W. C. C.,Zhang M.,Jia X., y CheungJ. T. M., "Finiteelementmodelingofthecontact interface 
betweentrans-tibial residual limbandprosthetic socket", MedEngPhys, vol. 26, no. 8, 655-662, 2004. [25] Agache P. G.,Monneur C.,Leveque J. L., yDe Rigal J., "MechanicalpropertiesandYoung'smodulusofhumanskin in vivo", Arch. Dermatol. Res, vol. 269, no. 3, 221-232, 1980.

[26] Diridollou S.,Patat F.,Gens F.,Vaillant L.,Black D.,Lagarde J. M.,Gall Y., y BersonM., "In vivo modelofthemechanicalpropertiesofthehumanskinundersuction", Skin Res Technol, vol. 6, no. 4, 214221, 2000.

[27] Hendriks F.M., Brokken D., van Eemeren J., Oomens C.W.J., Baaijens F.P.T., y Horsten J.B.A.M., "A numerical-experimental methodtocharacterizethe non-linear mechanicalbehaviourofhumanskin", Skin Res Technol, vol. 9, 274-283, 2003.

[28] Pailler-Mattei C.,Bec S., yZahouani H., "In vivo measurementsoftheelasticmechanicalpropertiesofhumanskinbyindentationtests", MedEngPhys, vol. 30, no. 5,599-606, 2008.

[29] Pulido H. G.,Salazar R. de la V.,Carrasco A. C., ySánchez M. O., "Análisis y diseño de experimentos". Mc Graw-Hill, 2008. 